\title{
Performance of a large area avalanche photodiode in a liquid xenon ionization and scintillation chamber
}

\author{
K. Ni ${ }^{\mathrm{a}, *}$, E. Aprile ${ }^{\mathrm{a}}$, D. Day ${ }^{\mathrm{a}, 1}$, K.L. Giboni ${ }^{\mathrm{a}}$, J.A.M. Lopes ${ }^{\mathrm{b}}$, \\ P. Majewski ${ }^{a}$, M. Yamashita ${ }^{a}$ \\ a Physics Department and Columbia Astrophysics Laboratory, Columbia University, New York, NY 10027, USA \\ ${ }^{\mathrm{b}}$ Departamento de Fisica, Universidade de Coimbra, P-3004-516 Coimbra, Portugal
}

Received 19 February 2005; received in revised form 25 May 2005; accepted 9 June 2005

Available online 13 July 2005

\begin{abstract}
Scintillation light produced in liquid xenon (LXe) by alpha particles, electrons and gamma-rays was detected with a large area avalanche photodiode (LAAPD) immersed in the liquid. The alpha scintillation yield was measured as a function of applied electric field. We estimate the quantum efficiency of the LAAPD to be $45 \%$. The best energy resolution from the light measurement at zero electric field is $7.5 \%(\sigma)$ for $976 \mathrm{keV}$ internal conversion electrons from

${ }^{207} \mathrm{Bi}$ and $2.6 \%(\sigma)$ for $5.5 \mathrm{MeV}$ alpha particles from ${ }^{241} \mathrm{Am}$. The detector used for these measurements was also operated as a gridded ionization chamber to measure the charge yield. We confirm that using a LAAPD in LXe does not introduce impurities which inhibit the drifting of free electrons.
\end{abstract}

(C) 2005 Elsevier B.V. All rights reserved.

Keywords: LAAPD; Liquid xenon; Dark matter

\section{Introduction}

In recent years, large area avalanche photodiodes (LAAPD) have been successfully applied as photodetectors for a variety of scintillators [1,2], including liquid xenon (LXe) [3]. LXe scintillation

\footnotetext{
*Corresponding author. Tel.: + 19145912825 ; fax: +19145914906.

E-mail address: nikx@astro.columbia.edu (K. Ni).

${ }^{1}$ Permanent address: University of Florida, Gainesville, FL 32611, Columbia Nevis Lab REU program participant (summer 2004).
}

light is in the VUV region, centered at $178 \mathrm{~nm}$ [4], which makes it difficult to be detected efficiently. Within the context of the R\&D for the XENON dark matter project [5], we are testing different photodetectors directly coupled to LXe, including UV sensitive photomultipliers (Hamamatsu R9288 and R8520), microchannel plate PMTs (Burle 85006), silicon photomultiplier (SiPM) [6] and LAAPDs from Advanced Photonix, Inc.(API). In this paper we report our first results on the detection of LXe scintillation with a $16 \mathrm{~mm}$ diameter LAAPD, mounted on a custom designed 
ceramic support. This kind of LAAPD is UV sensitive and has been previously successfully operated in high-pressure scintillation detectors [7]. The interest in LAAPDs for LXe is in their high quantum efficiency (QE) at $178 \mathrm{~nm}$, as originally reported by Ref. [3]. Their negligible radioactive contamination is also attractive for low background experiments based on LXe, such as direct dark matter searches (XENON [5]) and neutrinoless double beta decay searches (EXO [8]).

On the other hand, for a practical application of LAAPDs, especially for LXe based dark matter searches, several issues remain to be addressed. The primary concern is the capability of a light readout based on LAAPDs to achieve the low energy threshold of a few tens of $\mathrm{keV}$ required for high sensitivity. Other issues include long term stability of operation, impact on LXe purity, as well as readout complexity and cost. The experiments presented in this paper aimed at confirming the high QE for LXe scintillation reported in the literature, and at verifying the compatibility of LAAPDs immersed in the liquid with the requirement to detect tiny ionization signals, at the single electron level.

\section{Experimental set-up}

The $16 \mathrm{~mm}$ diameter, windowless LAAPD (API $\mathrm{S} / \mathrm{N}$ : 128-16-11) tested in this experiment was mounted inside a gridded ionization chamber, filled with high purity LXe. A photograph of the LAAPD and assembled electrodes is shown in Fig. 1. The cathode is a $6 \mathrm{~cm}$ diameter stainless steel plate with either a gamma ray source $\left({ }^{207} \mathrm{Bi}\right)$ or an alpha source $\left({ }^{241} \mathrm{Am}\right)$ deposited on its center. The shielding grid and the anode grid are made with stretched wires on stainless steel frames, with a thickness of about $0.8 \mathrm{~mm}$. The wire spacing is $2 \mathrm{~mm}$, and the wire diameter is $60 \mu \mathrm{m}$. The separation between cathode and grid is $1 \mathrm{~cm}$, which defines the maximum drift gap for ionization electrons. The grid to anode separation is $3 \mathrm{~mm}$. The electrodes spacing was maintained with rings made of PTFE, for its high reflectivity in the UV region [9]. The LAAPD, originally glued by API on a thin ceramic substrate, was mounted on a PTFE

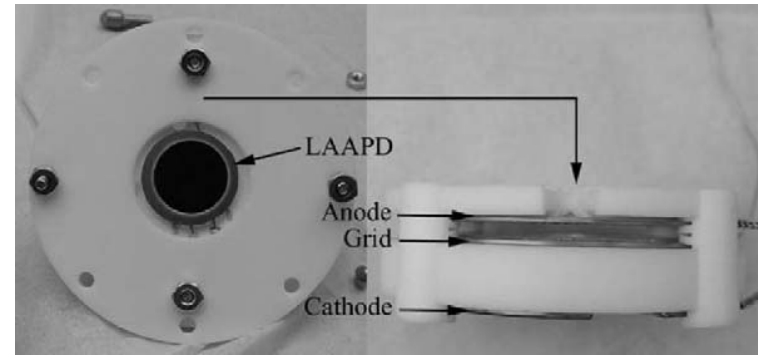

Fig. 1. Photograph of the assembled gridded ionization chamber electrodes, with the LAAPD mounted $6 \mathrm{~mm}$ above the anode grid. A PTFE plate is used to support the LAAPD above the anode grid. PTFE rings are used to maintain the electrodes spacing.

disk, facing the anode grid at a distance of $6 \mathrm{~mm}$ above. We note that the LAAPD had been exposed to air for several months prior to its use in LXe. Hermetic feedthroughs are used for cathode and grid HV lines and for anode signal readout via a charge sensitive amplifier. Additional feedthroughs are used to bias the LAAPD and to connect its output to a separate charge sensitive amplifier.

A schematic drawing of the detector system and electronics readout is shown in Fig. 2. The ionization electrons, which are created from gamma rays or alpha particles in the drift region, are drifted upward by the applied electric field, and are collected by a charge sensitive pre-amplifier (ClearPulse Model 580) connected to the anode. The scintillation light hits the LAAPD and produces photoelectrons, which are amplified by the avalanche process. The avalanche electrons are collected by an AmpTek 250 pre-amplifier. The charge and light signals, either from ClearPulse 580 or AmpTek A250, are fed into a low-noise shaping amplifier (ORTEC 450). For signals from the LAAPD, the shaping time of the amplifier was set at $0.25 \mu$ s, which was optimized for low dark noise and at the same time, sufficiently detecting the fast liquid xenon scintillation light, which has two scintillation components decaying at $4.3 \mathrm{~ns}$ and $22 \mathrm{~ns}$ [10]. The amplified signals are further fed into a PC-based multi-channel analyser (MCA) for spectroscopy analysis. Known test pulses are used to calibrate the DAQ system, for both light and charge signals. The capacitances in the preamplifiers were calibrated with a silicon detector. 


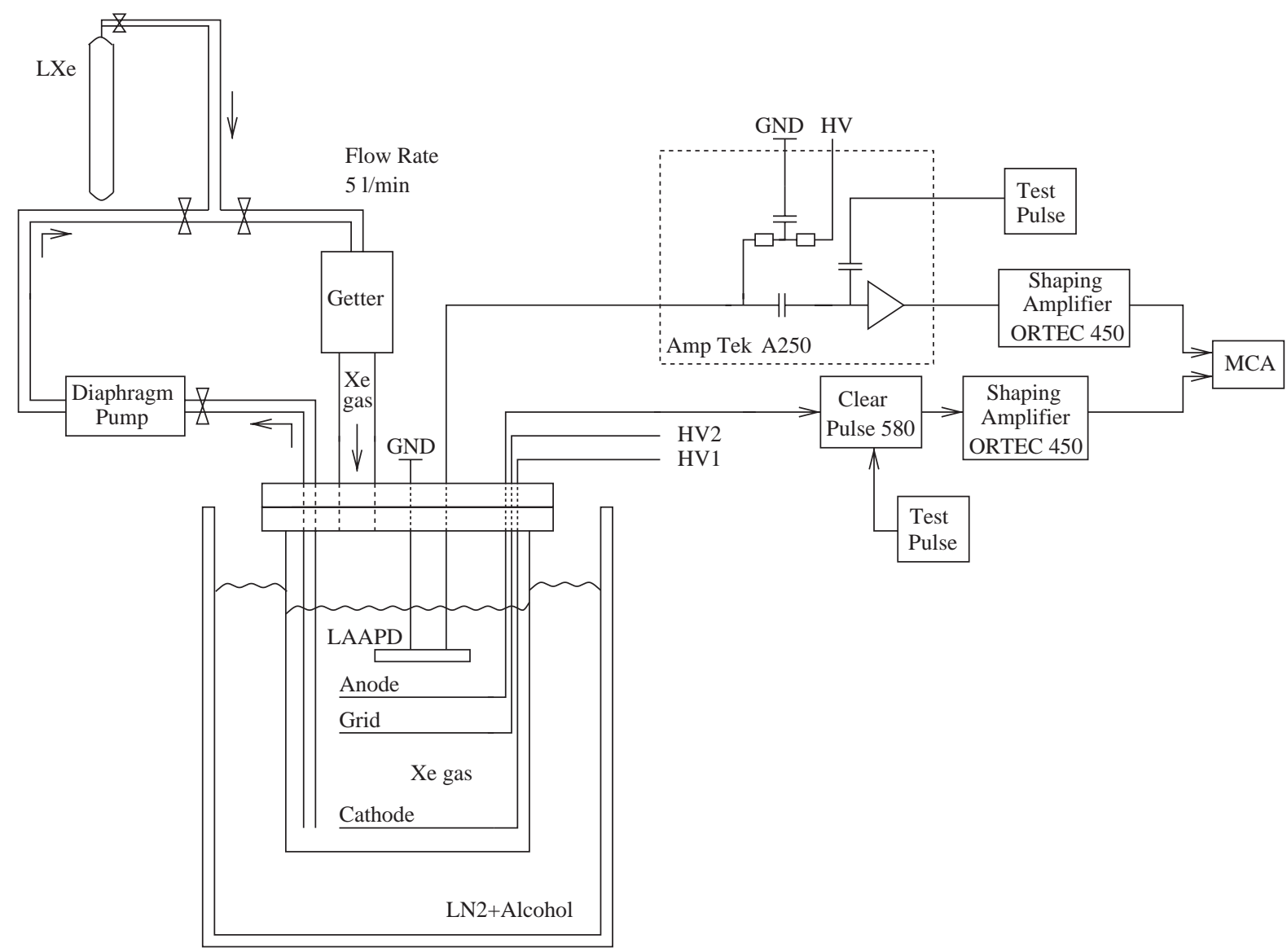

Fig. 2. DAQ schematics for the LAAPD setup for scintillation and ionization detection in liquid xenon. The top left part shows a simple schematics for the xenon gas recirculation and purification system.

We used an open bath cooling apparatus with a liquid nitrogen and alcohol mixture to condense the xenon gas. The vessel enclosing the assembled detector was filled with high purity LXe, covering completely the LAAPD. As shown in Fig. 2, we used a high temperature getter to purify Xe gas continuously via a diaphragm pump. The system, developed for XENON prototypes, is described in Ref. [11].

\section{Measurement and results}

\subsection{LAAPD gain}

The gain of the LAAPD was measured in a different set-up configuration, which optimizes the light collection efficiency by placing the LAAPD very close (about $3.5 \mathrm{~mm}$ ) to the source plate, at $-95^{\circ} \mathrm{C}$ (Fig. 3). The direct scintillation from a $5.5 \mathrm{MeV}$ alpha source $\left({ }^{241} \mathrm{Am}\right)$ was measured as a function of applied voltage on the LAAPD up to about $1500 \mathrm{~V}$. Unitary gain was determined from the average amplitude in the $300-500 \mathrm{~V}$ range [12]. At low voltages, the LAAPD capacity varies versus bias voltage. For gains above three and up to the highest gain, the capacity changes less than $1-1.5 \%$ [3]. In our setup, the dominant error for the gain measurement is from the temperature variations $\left( \pm 1^{\circ} \mathrm{C}\right)$. The error for the gains with this temperature variation is about $3 \%$. The typical reduction in applied voltage when reducing temperature at a constant gain was observed, corresponding to an 


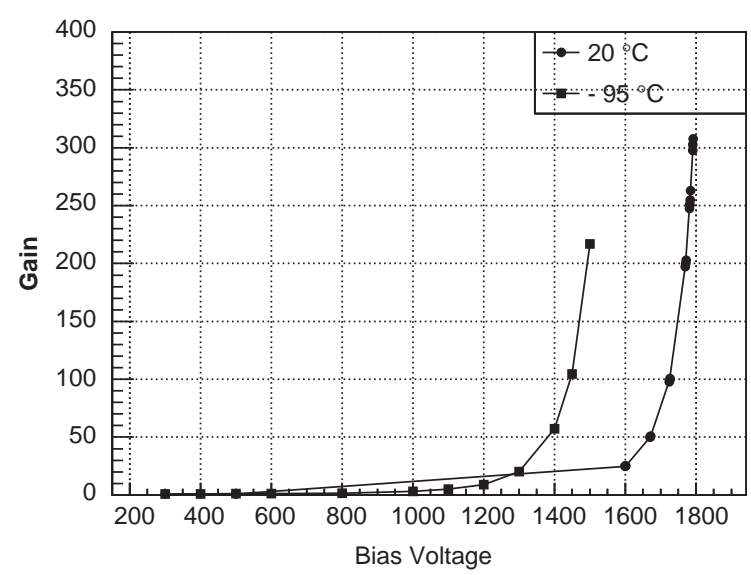

Fig. 3. LAAPD gain as a function of bias voltage measured at liquid xenon $\left(-95^{\circ} \mathrm{C}\right)$ and room temperature $\left(20^{\circ} \mathrm{C}\right)$. The data at room temperature is obtained from [23]. The gain with bias voltage of $1400 \mathrm{~V}$ is 57 at $-95^{\circ} \mathrm{C}$.

average voltage variation of about $2.4 \mathrm{~V} /{ }^{\circ} \mathrm{C}$ at a gain of 100 .

\subsection{Scintillation spectroscopy}

The scintillation light spectrum of the ${ }^{207} \mathrm{Bi}$ radiation in $\mathrm{LXe}$ was measured, as shown in Fig. 4. Due to the small liquid xenon sensitive volume, the energy from most of the $1064 \mathrm{keV}$ gamma rays of ${ }^{207} \mathrm{Bi}$ is not fully deposited. The second peak on the spectrum is mostly contributed by the $976 \mathrm{keV}$ internal conversion electrons. As the gamma rays interact at any point in the liquid xenon active volume, light collection in the LAAPD varies accordingly to the interaction position. Using PTFE reflectors, the variation in light collection can be reduced to less than $1 \%$, without compromising the energy resolution. The energy resolution for the $976 \mathrm{keV}$ electrons of ${ }^{207} \mathrm{Bi}$ is $7.5 \%(\sigma)$, which is comparable to the energy resolution obtained earlier by using a PMT in the same chamber with similar geometry [13]. The spectrum was accumulated at zero electric field to maximize light output from LXe.

To better evaluate the LAAPD performance in liquid xenon, larger amounts of scintillation light are necessary. The $5.5 \mathrm{MeV}$ alpha particles from ${ }^{241} \mathrm{Am}$ provide typically one order of magnitude

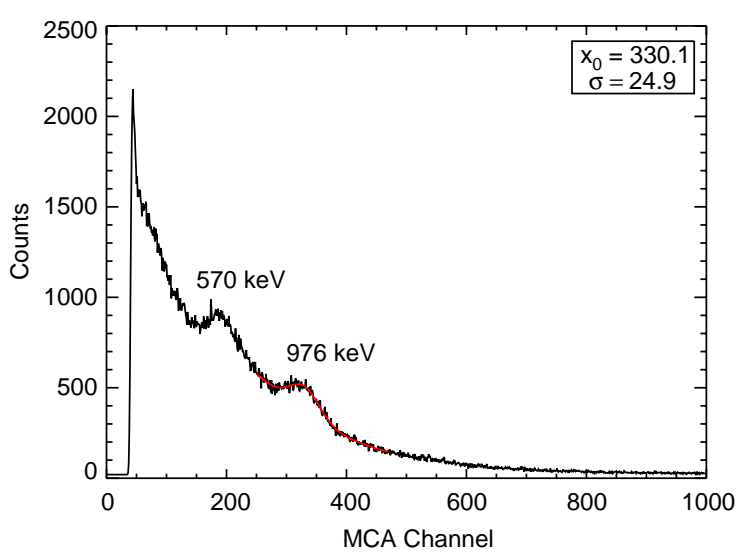

Fig. 4. Scintillation light spectrum from ${ }^{207} \mathrm{Bi}$ at zero electric field. LAAPD gain is about 146 and the temperature is $-95^{\circ} \mathrm{C}$. The peak of the $976 \mathrm{keV}$ electron line is fitted at about channel 330 , giving a resolution of $7.5 \%(\sigma)$. The $570 \mathrm{keV}$ gamma line can also be seen clearly at about channel 190 .

larger scintillation pulse, contributing to a clear scintillation light spectrum. Interaction of $\alpha$ particles in liquid xenon is very localized $(<0.1 \mathrm{~mm})$, which makes the geometrical variations of scintillation light collection negligible. To get the best possible energy resolution, the gain of LAAPD and shaping time on the amplifier were optimized and finally a gain of 57 was selected for this measurement as shown in Fig. 5. A very good energy resolution of $2.6 \%(\sigma)$ (with PTFE walls) and $3.0 \%(\sigma)$ (without PTFE walls) was obtained. To compare the performance of LAAPD with other photon detection devices, the alpha spectrum was also measured using a 2-in. diameter Hamamatsu R9288 PMT with less than 20\% QE at $178 \mathrm{~nm}$ wavelength. A value of $2.5 \%$ energy resolution was obtained. The energy resolution as a function of LAAPD gain was measured for both cases with and without PTFE wall. Fig. 6 shows the results in the case without PTFE wall.

The energy resolution $R$ as a function of LAAPD gain can be written as

$$
R=\sqrt{\left(\frac{N_{\text {noise }}}{N_{0} M}\right)^{2}+\frac{F}{N_{0}}+\delta_{\text {in }}^{2}} .
$$

Here $N_{\text {noise }}$ is the noise equivalent charge contributed from diode-amplifier system. The noise 

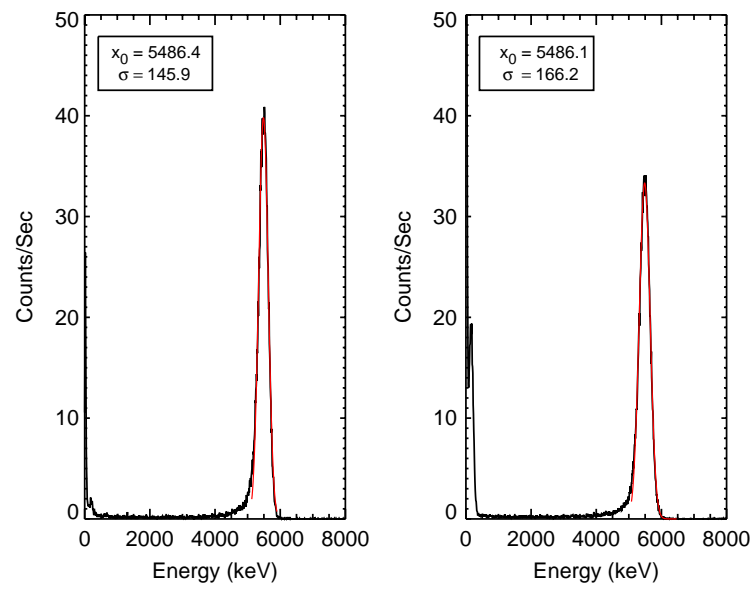

Fig. 5. ${ }^{241}$ Am scintillation light energy spectrum from $5.5 \mathrm{MeV}$ alpha particles detected by the LAAPD in LXe. The LXe temperature is $-95^{\circ} \mathrm{C}$ and LAAPD gain is 57 . The alpha peak is fitted with a Gaussian function. The energy resolution is $2.6 \%$ for the case with the PTFE wall (left) and $3.0 \%$ for the case without the PTFE wall (right). The peaks are normalized to the energy of the alpha particles.

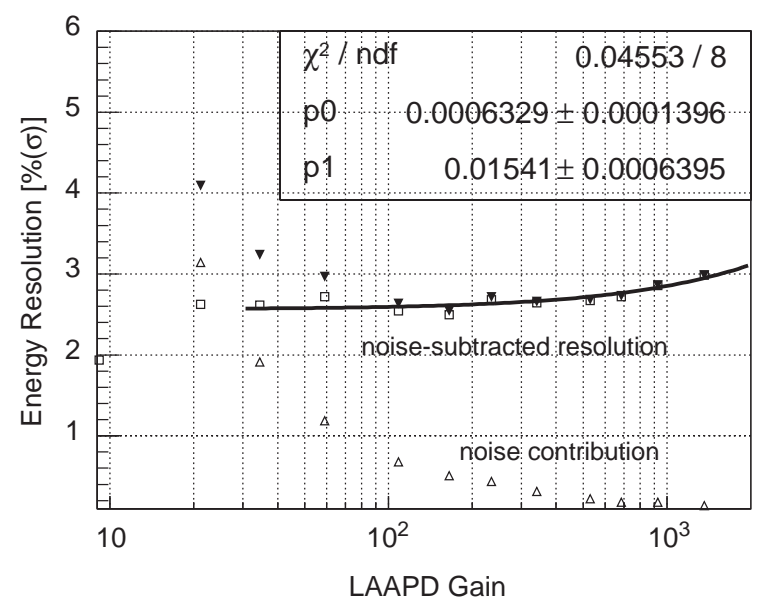

Fig. 6. Measured energy resolution (solid triangles) as a function of LAAPD gain. The measured dark noise contribution are shown in open-triangles. The open-squares are the noise-subtracted energy resolutions. With a fixed value of $F_{0}=1.7$, a fit to the noise-subtracted resolution gives $k=0.0006(\mathrm{p} 0)$ and $\delta_{\text {in }}=1.5 \%(\mathrm{p} 1)$.

contribution was measured from the width of peak distribution of a calibration test pulse. It is dependent on the shaping time of the spectroscopy amplifier, and decreases with higher LAAPD gain [14]. $N_{0}$ is the number of primary electron-hole pair produced from the scintillation light in the photodiode surface. $N_{0}$ was measured about 4050 in this setup. $M$ is the LAAPD gain. The term $F / N_{0}$ is from the fluctuations of the gain and statistics of the primary electron-hole pairs. It is inherent to the LAAPD, and is usually described in terms of excess noise factor $F$ [14]. $F$ can be approximately written as $F=F_{0}+k M$ for $M>30$, where $F_{0}$ and $k$ are parameters that describe the intrinsic property of the diode. $\delta_{\text {in }}$ is the intrinsic resolution of liquid xenon scintillation. The noise-subtracted energy resolution $R_{\mathrm{ns}}$ can be written as

$R_{\mathrm{ns}}=\sqrt{\frac{F_{0}+k M}{N_{0}}+\delta_{\mathrm{in}}^{2}}$.

By taken $F_{0}$ at a fixed value of 1.7 , which is the average value previously measured in Ref. [15], the noise-subtracted energy resolution was fit by equation (2). The fitting gives $k=0.0006 \pm$ 0.0001 and $\delta_{\text {in }}=1.5 \%$.

\section{Quantum efficiency}

The quantum efficiency $(\eta)$ of a LAAPD can be calculated by the following equation:

$\eta=\frac{N_{0}}{N_{\mathrm{p}}}=\frac{N_{\mathrm{d}} / M}{\alpha N_{\text {tot }}}$.

Here $N_{0}$ is the number of photoelectrons from the LAAPD, $N_{\mathrm{p}}$ is the number of photons reaching the LAAPD surface, $M$ is the LAAPD gain, $N_{\mathrm{d}}$ is the number of electron charges detected by the pre-amplifier and $N_{\text {tot }}$ is the total number of scintillation photons produced by an event. $N_{\text {tot }}$ is approximately equal to $E / W_{\mathrm{ph}}$, where $E$ is the energy of the event, and $W_{\mathrm{ph}}$ is the average energy required to produce a scintillation photon in liquid xenon. The $W_{\mathrm{ph}}$ values are $21.6 \mathrm{eV}$ [16] and $19.6 \mathrm{eV}$ [17] for gamma and alpha events respectively. $\alpha$ is the light collection efficiency, which is defined as the percentage of the total LXe light yield reaching the LAAPD surface. We have estimated the light collection efficiency by using a 
Table 1

LAAPD quantum efficiency from different measurements

\begin{tabular}{llllll}
\hline Measurement & PTFE & $\begin{array}{l}\text { Light Col. } \\
\text { Eff.(\%) }\end{array}$ & $\begin{array}{l}\text { Bias } \\
\text { V }\end{array}$ & $\begin{array}{l}\text { APD } \\
\text { gain }\end{array}$ & QE(\%) \\
\hline $976 \mathrm{keV} \mathrm{e}^{-}$ & With & $7.0 \pm 0.7$ & 1460 & 146 & $50 \pm 5$ \\
$5.5 \mathrm{MeV} \alpha$ & With & $7.0 \pm 0.7$ & 1400 & 57 & $39 \pm 4$ \\
$5.5 \mathrm{MeV} \alpha$ & Without & $3.7 \pm 0.4$ & 1400 & 57 & $39 \pm 4$ \\
\hline
\end{tabular}

light tracing simulation program in GEANT4 [18]. In the simulation, we assume a PTFE reflectivity between $90 \%$ and $95 \%$ [9], the UV light absorption length of $100 \mathrm{~cm} \mathrm{[19],} \mathrm{and} \mathrm{UV} \mathrm{light} \mathrm{Rayleigh}$ scattering lenght of $30 \mathrm{~cm} \mathrm{[20]} \mathrm{in} \mathrm{liquid} \mathrm{xenon.}$

The estimated light collection efficiency from the simulation is $(7.0 \pm 0.7) \%$ for the structure with $1 \mathrm{~cm}$ thick PTFE wall between the cathode and grid. The error indicates the different reflectivity values used in the simulations. In the case of no PTFE walls, the light collection efficiency was estimated to be $(3.7 \pm 0.4) \%$.

From the above considerations, we estimated the QE of the LAAPD for different measurements, which are shown in Table 1. The main uncertainty is from the estimation of the light collection efficiency. The different values obtained from electron and alpha events may be due to the uncertainty in $W_{\text {ph }}$ and the LAAPD gain values. In conclusion, we use the average of the $\mathrm{QE}$ values from the electron and alpha measurements, which is $(45 \pm 5) \%$.

\section{Field dependence of light yield}

The liquid xenon scintillation light yield depends on the strength of the applied electric field. This dependence was measured in the current setup with the LAAPD. For comparison, we also measured this field dependence with a Hamamatsu R9288 PMT immersed in liquid xenon, in the same chamber (Fig. 7).

The LAAPD gain varies considerably with temperature, and our simple alcohol- $\mathrm{LN}_{2}$ cooling bath does not keep the liquid temperature stable enough to avoid temperature dependent gain variations. The gain of the PMT is not much

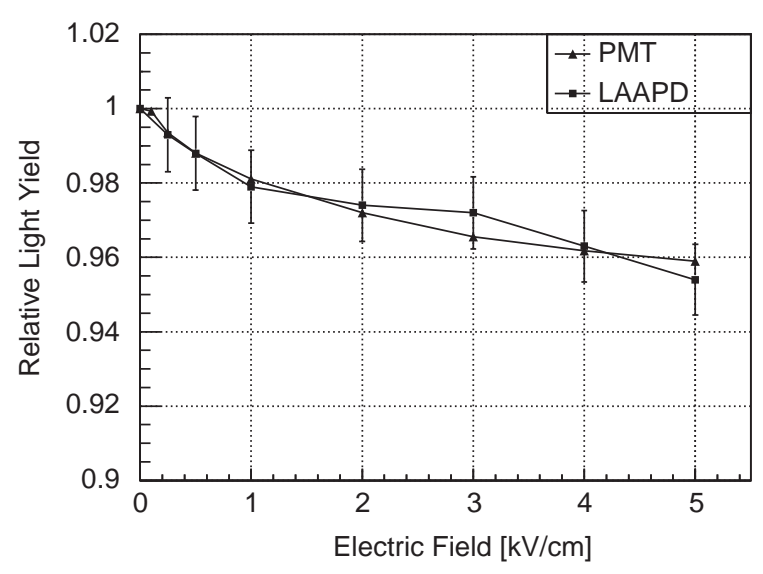

Fig. 7. Field dependence of light yield for ${ }^{241} \mathrm{Am} 5.5 \mathrm{MeV}$ alpha particles. The measurements were carried out with either a PMT or a LAAPD, immersed in liquid xenon at around $-95^{\circ} \mathrm{C}$. In the LAAPD measurement, the temperature was controlled with less than $0.5^{\circ} \mathrm{C}$ variations. The error bars $(1 \%)$ on the LAAPD data are from the LAAPD gain variation due to temperature fluctuations.

affected by such small temperature fluctuations. The data from the LAAPD and the PMT are in good agreement, but the result with the LAAPD has more fluctuations due to its acute temperature dependence, which is obvious from the curve.

\section{Impact of LAAPD on LXe purity}

One challenge involving photon detection devices immersed in liquid xenon is their compatibility with the high purity required for electron drift, if a combined charge and light readout is implemented. Our experience with LXe detectors shows that the light yield of LXe is not very sensitive to the purity level, unlike the charge yield. Many efforts have been made in the past to detect both ionization and scintillation, using PMTs, in liquid xenon [21]. Currently we have developed a xenon recirculation and purification system [11], which continuously removes impurities from the liquid xenon during experiment. Depending on the size of the detector, we can achieve a sufficient purity level for drifting ionization electrons within two to tens of hours. During the first experiment with the LAAPD immersed in the LXe, we used 


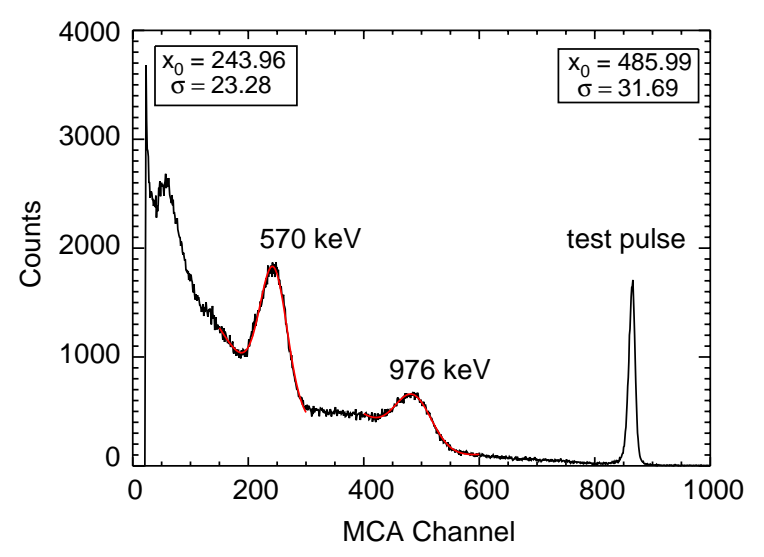

Fig. 8. Ionization spectrum from ${ }^{207} \mathrm{Bi}$ radiation in liquid xenon at $1 \mathrm{kV} / \mathrm{cm}$ drift field with an LAAPD immersed in the liquid. The peaks are from $570 \mathrm{keV}$ gamma rays (channel 244) and $976 \mathrm{keV}$ internal conversion electrons (channel 486). The sharp peak on the right of the charge spectrum is from a known test pulse. Based on the peak values, we estimated the charge collection at about $75 \%$, by assuming the average energy needed to produce an electron ion pair in liquid xenon is $15.6 \mathrm{eV}$ [24]. The energy resolution for the $570 \mathrm{keV}$ gamma rays and $976 \mathrm{keV}$ electron peaks are $9.5 \%$ and $6.5 \%$ separately.

this recirculation system to purify the xenon, continuously. We measured the $976 \mathrm{keV}$ peak position of the ${ }^{207} \mathrm{Bi}$ to monitor the charge collection. Within a few hours of recirculation and purification, we achieved a high charge collection of $75 \%$. The ionization spectrum of ${ }^{207} \mathrm{Bi}$ at $1 \mathrm{kV} / \mathrm{cm}$ drift field shown in Fig. 8 is comparable to that measured in a liquid xenon ionization chamber with an external PMT [22]. From the second experiment, we observed the same level of charge collection even without using the recirculation system, which indicates that the LAAPD is clean and does not bring any impurities into the liquid xenon.

\section{Conclusion}

In this paper, we have demonstrated the operation of a LAAPD in liquid xenon to detect scintillation light from gamma rays and alpha particles. We have achieved very good energy resolution for alpha particles, from liquid xenon scintillation light detected by a LAAPD. The inferred value of the QE is $45 \%$. It is lower than that previously reported in Ref. [3], which used a different type of LAAPD (DUV) with enhanced quantum efficiency for the xenon scintillation light. We intend to pursue the same type of LAAPD to improve our results.

The gain of the LAAPD is much lower than that of PMTs. Using an external amplification system limits the energy detection threshold, which is very crucial for a sensitive dark matter detector. The scintillation light from a WIMP recoil of a few tens of $\mathrm{keV}$ energy may produce not enough light to be detected by the LAAPD. On the other hand, properties of LAAPDs, such as their compact size, very high $\mathrm{QE}$, and compatibility with low radioactive background and high LXe purity requirements, make them attractive for applications of LXe detectors in particle physics, medical imaging and astrophysics.

\section{Acknowledgements}

This work was supported by a grant from the National Science Foundation to the Columbia Astrophysics Laboratory (Grant No. PHY-0201740). One of the authors (P. Majewski) acknowledges the support by the North Atlantic Treaty Organization under a grant awarded in 2003.

\section{References}

[1] J.A.M. Lopes, et al., IEEE Trans. Nucl. Sci. NS-48 (3) (2001) 312.

[2] M. Moszynski, et al., Nucl. Instr. and Meth. A 497 (2003) 226.

[3] V.N. Solovov, et al., Nucl. Instr. and Meth. A 488 (2002) 572.

[4] J. Jortner, et al., J. Chem. Phys. 42 (1965) 4250.

[5] E. Aprile, et al., astro-ph/0207670.

[6] P. Shagin, et al., physics/0501002, Nucl. Instr. and Meth. A, accepted for publication.

[7] A.I. Bolozdynya, R.A. Austin, Proc. SPIE 5540 (2004) 206;

A. Arodzero, et al., IEEE Trans. Nucl. Sci. NS-51 (2) (2004) 322.

[8] EXO Experiment, see online http://www-project.slac.stanford.edu/exo/.

[9] M. Yamashita, et al., Nucl. Instr. and Meth. A 535 (2004) 692. 
[10] A. Hitachi, et al., Phys. Rev. B 27 (1983) 5279.

[11] E. Aprile, et al., New Astronomy Rev. 49 (2005) 289 astroph/0407575.

[12] L.M.P. Fernandes, et al., IEEE Trans. Nucl. Sci. NS-51 (4) (2004) 1575

[13] E. Aprile, et al., IEEE Trans. Nucl. Sci. NS-51 (5) (2004) 1986.

[14] M. Moszynski, et al., Nucl. Instr. and Meth. A 485 (2002) 504.

[15] L.M.P. Fernandes, et al., Nucl. Instr. and Meth. A 531 (2004) 566.

[16] T. Doke, et al., Jpn. J. Appl. Phys. 41 (2002) 1538.
[17] T. Doke, et al., Nucl. Instr. and Meth. A 420 (1999) 62.

[18] S. Agostinelli, et al., Nucl. Instr. and Meth. A 506 (2003) 250.

[19] A. Baldini, et al., Nucl. Instr. and Meth. A 545 (2005) 753.

[20] G.M. Seidel, et al., Nucl. Instr. and Meth. A 489 (2002) 189.

[21] E. Aprile, et al., IEEE Trans. Nucl. Sci. NS-50 (5) (2003) 1303.

[22] E. Aprile, et al., Nucl. Instr. and Meth. A 302 (1991) 177.

[23] A.I. Bolozdynya, private communication.

[24] T. Takahashi, et al., Phys. Rev. A 12 (1975) 1771. 